\title{
Evolution of Rotating AGB Stars and the s-Process Nucleosynthesis
}

\author{
Lionel Siess ${ }^{1}$, Stéphane Goriely ${ }^{1}$ and Norbert Langer ${ }^{2}$ \\ ${ }^{1}$ Institut d'Astronomie et d'Astrophysique, Université Libre de Bruxelles, CP 226, \\ B-1050 Brussels, Belgium \\ siess@astro.ulb.ac.be \\ ${ }^{2}$ Astronomical Institute, Universiteit Utrecht, P.O. Box 80000, NL-3508 TA Utrecht, The Netherlands
}

Received 2003 May 2, accepted 2003 September 2

\begin{abstract}
We present new results on the evolution and nucleosynthesis in rotating AGB stars. We analyse the role of the gradient of mean molecular weight in the mixing process and show that neglecting this component induces a potentially strong third dredge-up. We also quantify the impact of rotation on the structure and conclude that the effects of rotation (1) mainly concern the inner, fast rotating regions of the stars and (2) are relatively weak as long as rotational mixing does not induce a deep third dredge-up. We also focus our investigations on the s-process nucleosynthesis and show that rotational mixing tends to inhibit the production of s-elements. This results from the contamination of the ${ }^{13} \mathrm{C}$-rich layers responsible for the neutron production by the poisonous ${ }^{14} \mathrm{~N}$. Our calculations also indicate that the distribution of s-process elements depends sensitively on the magnitude of the diffusion coefficient. These results suggest that rotational mixing is not the main mechanism responsible for the production of s-elements in AGB stars, but that it can influence, and in particular reduce, the final enrichment in s-elements.
\end{abstract}

Keywords: stars: AGB and post-AGB — stars: rotation — nuclear reactions, nucleosynthesis, abundances

\section{Introduction}

In AGB stars, the synthesis of s-elements is mainly due to the release of neutrons by ${ }^{13} \mathrm{C}(\alpha, \mathrm{n}){ }^{16} \mathrm{O}$ at low temperature $\left(T \sim 10^{8} \mathrm{~K}\right)$ and marginally by ${ }^{22} \mathrm{Ne}(\alpha, \mathrm{n}){ }^{25} \mathrm{Mg}$ at the bottom of the hot convective pulse where the temperature $T$ exceeds $3 \times 10^{8} \mathrm{~K}$. The formation of the ${ }^{13} \mathrm{C}$-rich layers responsible for the production of neutrons has long been a debated issue but a general consensus has now been reached that some kind of extra mixing must take place at the base of the convective envelope at the time of the third dredge-up (3DUP). In this framework, protons mix with the carbon left behind after the thermal pulse and their burning via ${ }^{12} \mathrm{C}(\mathrm{p}, \gamma){ }^{13} \mathrm{~N}\left(\beta^{+}\right)^{13} \mathrm{C}$ lead to the production of the neutron seeds. However, large uncertainties still remain on the exact nature of the mixing processes and the most popular scenario implies convective overshoot (Herwig, Blöcker, \& Schönberne 1997; Herwig 2000), for example by gravity waves (Denissenkov \& Tout 2003), or rotationally induced mixing (Langer et al. 1999).

In this paper, we focus on the rotationally induced mixing and analyse how it affects the s-process nucleosynthesis. But before going ahead, let us briefly recall the main characteristics of the above mentioned processes. The overshoot model is a simple, parametric extension of the convective border beyond the traditional limit defined by the standard mixing length theory to account for the non-vanishing velocity of the convective cells at the radiative-convective interface. Contrary to the early studies of overshoot which mainly focus on main-sequence stars, the current implementation of this mixing process considers a depth and time-dependent description motivated by hydrodynamical simulations (Freytag, Ludwig, \& Steffen 1996). The gravity wave model is also linked to the presence of a convective zone and is based on the idea that the internal waves arising from a perturbation of the gravitational field will generate turbulence at the base of the convective envelope, thus extending the convective border outside its standard limits. As in the overshoot model, extra mixing is here treated as a diffusive phenomenon taking place at the base of the convective zone. On the other hand, the rotationally induced mixing is induced by the development of shear instabilities in regions of strong differential rotation. By nature these mechanisms are very different, and in particular it is worth emphasising that the overshoot and gravity waves models are intimately connected to the location of the convective zone. When the convective zone moves, the mixing zone also moves. This is not the case for the rotationally induced mixing. In AGB stars, the differential rotation that establishes at the base of the convective envelope at the time of the 3DUP persists long after the envelope has receded. For this reason, the rotational mixing is not reduced to a fast injection of protons within the C-rich layers at the time of the 3DUP, but also includes a continuous and slow chemical mixing between the different layers contaminated by the proton injection during a large fraction of the interpulse period.

Our study is based on post-processing calculations of the full nucleosynthesis inside rotating AGB stars. We use the structure profiles generated by the stellar evolution code (temperature, density and diffusion coefficients) as input to our nuclear code and follow the nucleosynthesis during the full interpulse phase. In the next section, we briefly describe the nuclear code. Then in Sect. 3 we analyse the evolutionary and structural differences between rotating and non-rotating models and the role of the mean molecular weight in this context. Finally in Sect. 4 we present our results of the s-process nucleosynthesis 
in rotating AGB models that experience the 3DUP and conclude with Sect. 5 .

\section{A New Nucleosynthesis Code}

When the mixing timescale is of the order of the nuclear burning timescale, the assumption of decoupling the nucleosynthesis and the transport equations becomes invalid and one must solve the full, coupled, set of equations. In the specific scenario considered here, i.e. rotating AGB stars, this situation is found at the time of radiative ${ }^{13} \mathrm{C}$-burning when the timescale for $\alpha$ captures on ${ }^{13} \mathrm{C}$ (a few times $10^{3} \mathrm{yr}$ at $T \sim 10^{8} \mathrm{~K}$ ) is comparable to the rotational diffusion timescale. Aware of this constraint, we designed a new nuclear code which couples the diffusion and nucleosynthesis equations. For each species $i$ the evolution of the molar abundance $Y_{i}$ is given by

$$
\frac{\partial Y_{i}}{\partial t}=\text { nuclear terms }+\frac{\partial}{\partial m_{r}}\left[\left(\frac{\partial m_{r}}{\partial r}\right)^{2} D_{r} \frac{\partial Y_{i}}{\partial m_{r}}\right],
$$

where $D_{r}=D_{\text {conv }}+D_{\text {rot }}$ is the sum of the Eulerian convective and rotational diffusion coefficients, respectively.

These equations are linearised and solved by the DASPK package (Brown, Hindmarsh, \& Petzold 1994, 1998) which uses a robust and efficient implicit method to invert large matrices. It makes use of pre-conditioners and is based on the Krylov method. The equations are written in a Lagrangian scheme. The initial chemical profiles injected in the nuclear code are those from the stellar evolution code at the time of the 3DUP. The thermodynamic conditions (temperature, density) are interpolated from the stellar models at the given time and mass coordinate. We also adopt a constant mass grid during the entire computation. For the diffusion problem we are dealing with, the convective envelope is assumed to represent an infinite reservoir and the boundary condition set in radiative regions allows for the matter to flow through this grid point. The mixing zone is typically described by $100-200$ shells and covers a mass range of the order of $10^{-4} \mathrm{M}_{\odot}$.

The input physics of the nuclear code is the same as the one described in Goriely \& Mowlavi (2000) and Goriely \& Siess (2001). Briefly, the nuclear reaction network includes about 547 nuclei up to Po with all relevant nuclear (n-, p-, $\alpha$-captures), weak (electron captures, $\beta$ decays) and electromagnetic (photodisintegration) interactions. Nuclear reaction rates are taken from the Nuclear Astrophysics Library of the Brussels University (available at http://www-astro.ulb.ac.be), which includes the latest experimental cross sections and calculated rates within the statistical Hauser-Feshbach model (Jorissen \& Goriely 2001). In particular, all the charged-particle-induced reaction rates of relevance in the $\mathrm{H}$ - and He-burning calculations presented here are taken from the European compilation of experimental rates, known as NACRE (Angulo et al. 1999). The $\beta$-decay and electron capture rates in stellar conditions are taken from Takahashi \& Yokoi (1987) with the update of Goriely (1999).

\section{Evolution of Rotating AGB Stars}

The treatment of rotational mixing, as well as the physics of the stellar evolution code, has been presented in detail in Heger et al. (2000) and we refer the reader to this reference for ampler details. The initial stellar model is for a star of a $3 \mathrm{M}_{\odot}, Z=0.02$ and initial equatorial velocity of $250 \mathrm{~km} \mathrm{~s}^{-1}$. It was analysed by Langer et al. (1999) and has been evolved to the 29th thermal pulse from which we start our study. In order to illustrate the effects of rotation on the evolution and structure of AGB stars, we run a series of three tests: a non rotating model (model A) and two different rotating AGB stars characterised by a shallow (model B) and deep (model C) 3DUP. The 3DUP in model $\mathrm{C}$ is initiated by omitting the effects of mean molecular weight in the treatment of rotational mixing $\left(f_{\mu}=0\right.$ instead of 0.05 in model B, see Sect. 3.2). Based on these data, we analyse the structural effects of rotation on the 3DUP and the chemical mixing.

\subsection{Stellar Evolution with Rotation}

Figure 1 depicts the structural evolution of a $3 \mathrm{M}_{\odot}$ model that experiences a deep 3DUP. The grey scale refers to the magnitude of the diffusion coefficient with the same coding as in Langer et al. (1999): the lighter the grey, the higher the rotational diffusion coefficient.

During the thermal pulse, the convective instability deepens and reaches into the inner and fast rotating layers of the CO core (see also Herwig, Lugaro, \& Langer 2003). Angular momentum is dredged up and redistributed over the convective zone. Eventually, when the instability decays, a fast rotating region is left in place of the pulse which is subsequently subject to structural readjustments. In the contracting regions, the rotational velocity increases and in expanding regions it decreases. In addition to the building up of this differential rotation, the end of the pulse is accompanied by a deepening of the convective envelope. As a result of the 3DUP, a strong differential rotation is established at the base of the convective envelope which is responsible for mixing.

During the interpulse phase (Fig. 2) which lasts for $3.2 \times 10^{4} \mathrm{yr}$, angular momentum is transported and the Eulerian diffusion coefficient $D_{r}$ is significantly reduced. In $\sim 2000 \mathrm{yr}, D_{r}$ has decreased by 3 orders of magnitude in the layers where protons were injected, i.e. at the bottom of the convective envelope at the time of the 3DUP, but some differential rotation still remains in this thin region of $\sim 10^{-4} \mathrm{M}_{\odot}$. Contraction in the intershell is important and $\partial m_{r} / \partial r$ is large in Eq. 1. As a consequence, the effective Lagrangian diffusion coefficient $\left[D_{m}=\left(\partial m_{r} / \partial r\right)^{2} D_{r}\right]$ remains relatively large and an efficient mixing is maintained during most of the interpulse period in this region (Herwig et al. 2003). In particular, it leads to the migration of ${ }^{14} \mathrm{~N}$ into the ${ }^{13} \mathrm{C}$ layer with dramatic consequences on the s-process (see next section).

\subsection{The Role of the $\mu$-Gradient}

The third dredge-up is responsible for the formation of carbon stars and is required for the s-process nucleosynthesis 


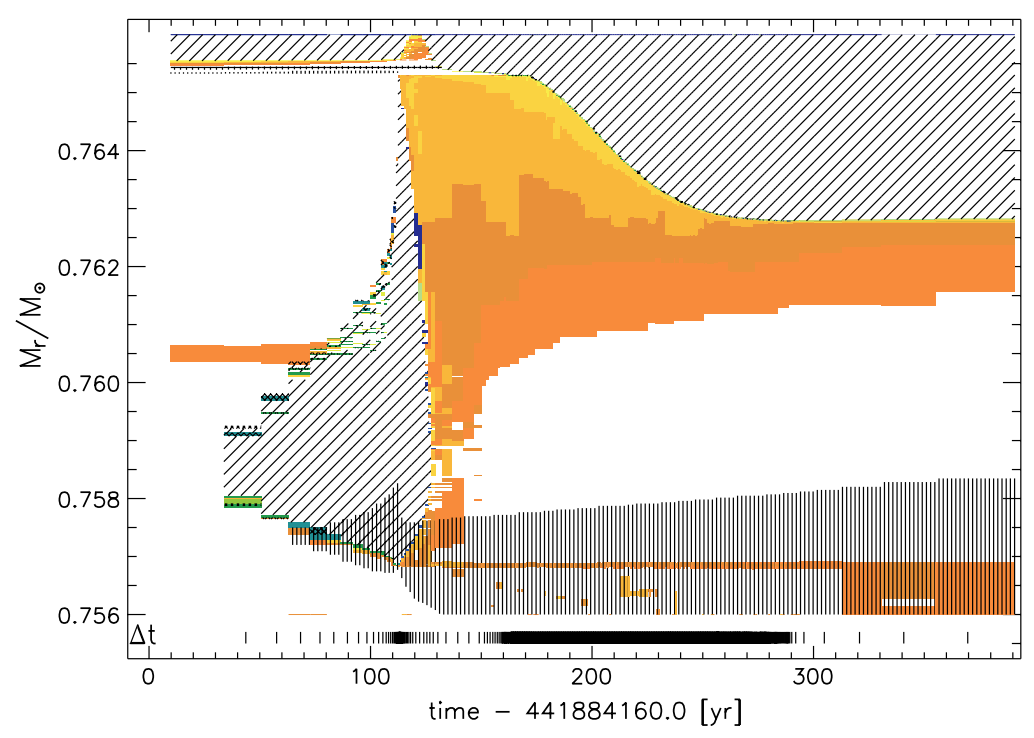

Figure 1 Structural evolution of a $3 \mathrm{M}_{\odot}$ star during the 29th thermal pulse. The model was computed with $f_{\mu}=0$ and shows a deep 3DUP. The convective zones are represented by shaded areas and the grey scale corresponds to magnitude of the Eulerian diffusion coefficient $\left(D_{r}\right)$ with the following coding: the lighter the grey, the larger the diffusion coefficient, typically at the base of the convective envelope. The vertical hatching represents the nuclear burning regions.

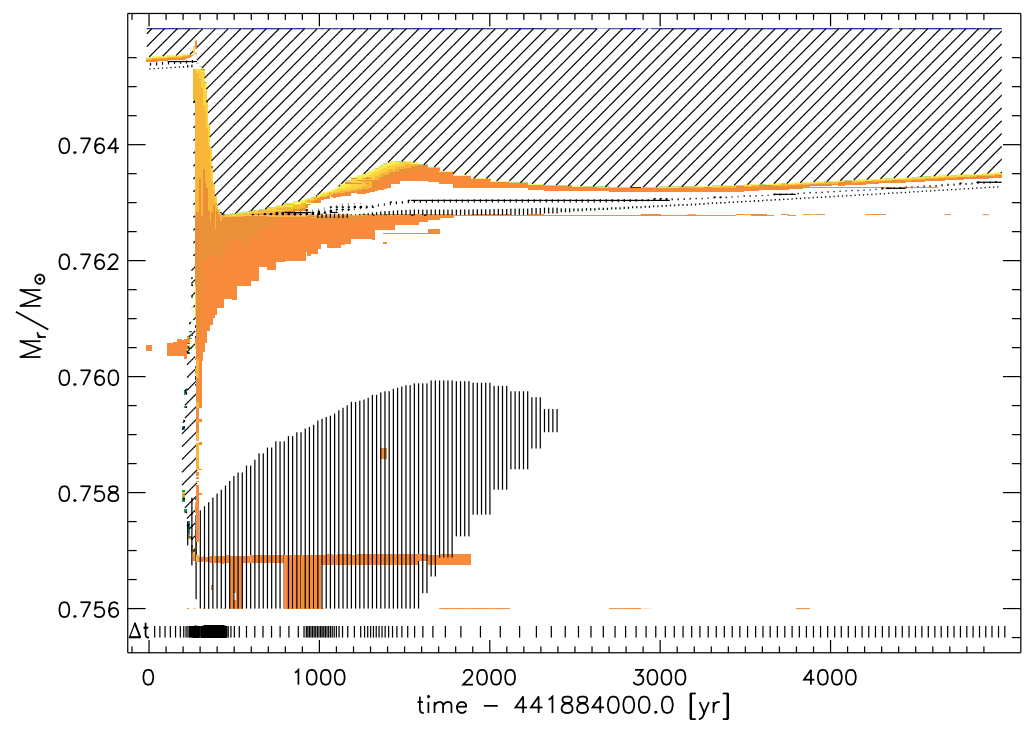

Figure 2 Structural evolution of a $3 \mathrm{M}_{\odot}$ star during the interpulse phase following the 29th pulse. The coding is the same as in Figure 1.

to take place. Modelling the 3DUP has been revealed to be critically dependent on the numerical scheme and physical processes acting at the base of the convective envelope. In particular it was shown that the strength of dredgeup depends on the space and time resolutions (Straniero et al. 1997), on the algorithm used to define the convective boundaries (e.g. Frost \& Lattanzio 1996), and is clearly favoured if some overshoot is included at the base of the convective envelope (Herwig 2000). Rotation also induces mixing but its efficiency to trigger the 3DUP event critically depends on the parameter $f_{\mu}$. This parameter was introduced to test the sensitivity of the rotational diffusion coefficient to the gradient of mean molecular weight by simply substituting $\nabla_{\mu}$ with $f_{\mu} \nabla_{\mu}$. As discussed in Heger et al. (2000), the calibration of $f_{\mu}$ is difficult, especially because other instabilities can develop and compensate for its effects. However, in the context of AGB stars, its impact on the structure appears to be substantial as illustrated by comparing the properties of models $\mathrm{B}\left(f_{\mu}=0.05\right)$ and $\mathrm{C}$ $\left(f_{\mu}=0\right)$ in Table 1. Setting $f_{\mu}=0$ increases the magnitude of the diffusion coefficient and at the time of the 3DUP, below the convective envelope, it is more than one order of magnitude larger in model $\mathrm{C}$ than in model $\mathrm{B}$. Under these circumstances, and similarly in other studies involving additional extra-mixing such as overshoot, the deepening of the convective envelope is substantially 
Table 1. Comparison of selected quantities between the non-rotating (A) and the rotating models (B and $\mathrm{C}$ ) with $f_{\mu}=0.05$ (B) and $f_{\mu}=0(\mathrm{C})$. The angular velocity is $\Omega, f_{\mu}$ is the parameter controlling the effects of the mean molecular weight, $M_{\text {pulse }}$ the mass extent of the pulse, $\Delta t_{\text {pulse }}$ its duration, $L_{\mathrm{He}}^{\max }$ the maximum He luminosity reached during the thermal instability, $\Delta t_{\text {interp }}$ the duration of the interpulse phase and $\lambda_{\text {DUP }}$ the dredge-up parameter

\begin{tabular}{|c|c|c|c|c|c|c|}
\hline \multirow[t]{2}{*}{ Model } & \multicolumn{3}{|c|}{ Pulse \# 29} & \multicolumn{3}{|c|}{ Pulse \# 30} \\
\hline & A & B & $\mathrm{C}$ & A & $\mathrm{B}$ & $\mathrm{C}$ \\
\hline$\Omega\left(\mathrm{km} \mathrm{s}^{-1}\right)$ & 0 & 250 & 250 & 0 & 250 & 250 \\
\hline$f_{\mu}$ & 0 & 0.05 & 0 & 0 & 0.05 & 0 \\
\hline$M_{\text {pulse }}\left(10^{-4} \mathrm{M}_{\odot}\right)$ & 8.4 & 8.5 & 8.5 & 8.2 & 8.4 & 8.9 \\
\hline$\Delta t_{\text {pulse }}(\mathrm{yr})$ & 97 & 98 & 98 & 89 & 102 & 105 \\
\hline$L_{\mathrm{He}}^{\max }\left(10^{7} \mathrm{~L}_{\odot}\right)$ & 5.69 & 5.89 & 5.83 & 6.52 & 6.76 & 11.35 \\
\hline$\Delta t_{\text {interp }}(\mathrm{yr})$ & 28760 & 29200 & 32300 & 27700 & 28100 & 33250 \\
\hline$\lambda_{\text {DUP }}$ & 0 & 0.043 & 0.53 & 0.002 & 0.064 & 0.61 \\
\hline
\end{tabular}
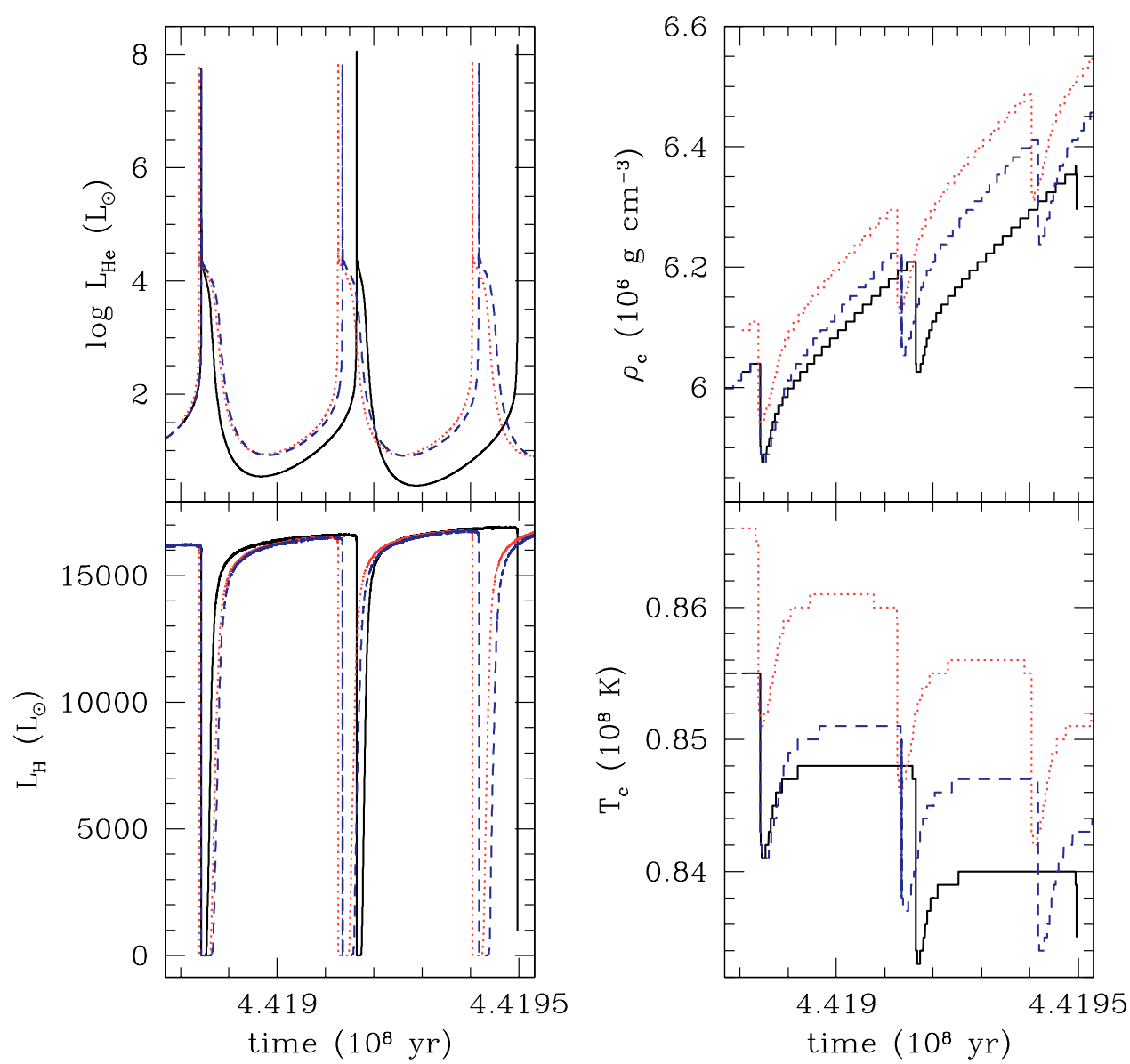

Figure 3 Evolution of the central density $\left(\rho_{c}\right)$ and temperature $\left(T_{c}\right)$ and nuclear luminosities due to $\mathrm{H}$ - and He-burning in the rotating models with $f_{\mu}=0$ (solid) and $f_{\mu}=0.05$ (dashed) and in the non-rotating (dotted) models of a $3 \mathrm{M}_{\odot}$ AGB star of solar metallicity. The evolution is shown from pulse number 29 to 31 .

enhanced. The DUP parameter $\lambda$ increases suddenly from 0.043 to 0.53 and in the next pulse an even larger value of 0.61 is reached in model $\mathrm{C}$. This strong effect results mainly from the fact that extra-mixing lowers the chemical gradient at the base of the envelope. The jump in the opacity coefficient, which depends sensitively on the chemical composition, is then reduced and the neutrality of the radiative and adiabatic gradients $\left(\nabla_{\mathrm{rad}}=\nabla_{\mathrm{ad}}\right)$ is almost met at the convective border. As discussed, for example, in Mowlavi (1999) and Pols \& Tout (2000), this situation leads to a deeper 3DUP and our larger value of the diffusion coefficient in model $\mathrm{C}$ supports this conclusion.

This tendency of a rapidly increasing $\lambda$ was pointed out by Karakas, Lattanzio, \& Pols (2002) who showed that in a few pulses (4 or more) an asymptotic regime can be reached for a $3 \mathrm{M}_{\odot}$ star. Under these circumstances we may expect that neglecting the $\mu$-gradient effect during the AGB phase would lead to the rapid development of 
deep 3DUPs that could possibly give rise to the formation of carbon stars at relatively low luminosities.

In Figure 3 we depict the evolution of the central temperature, density and nuclear luminosities due to $\mathrm{He}$ - and $\mathrm{H}$-burning. In model $\mathrm{C}$, the dredge-up rapidly quenches the He-shell flash and the presence of a deep convective envelope contributes to the efficient removal of energy from the intershell. As a consequence, less heat is transferred to the degenerate core and this explains why the central temperature in model $\mathrm{C}$ is smaller than in model B. The 3DUP also modifies the temperature stratification of the intershell and the resulting cooler structure postpones the development of the thermal instability which ignites $\sim 3500$ yr later in model $\mathrm{C}$ than in model B. Because of this delay, at the time of $\mathrm{He}$ ignition the core mass is slightly larger in model $\mathrm{C}$ and the pulse stronger. In addition, the more efficient mixing at the base of the convective pulse in model $\mathrm{C}$ contributes to burning $\mathrm{He}$ at higher temperatures and to increasing the strength of the pulse (e.g. Herwig 2000) which in turn will also favour a deeper 3DUP.

\subsection{The Impact of Rotation on the Structure}

In order to quantify the impact of rotation on the structure of AGB stars, we artificially set the rotational profile to zero in our initial model and followed the two subsequent pulse-interpulse phases. We are aware that this is not consistent but it is indicative of the effects of rotation alone. To disentangle effects induced by 3DUP, we will focus on the comparison between model A and model B.

The effect of rotation is two-fold: on one hand the stellar equations are modified to account for the centrifugal forces and on the other hand, rotation induces mixing which can significantly affect the structure. In the rotating model, centrifugal forces sustain the structure and lead to lower central temperatures and densities. A detailed study of the energetics indicates that the $\mathrm{H}$ luminosity (which provides most of the energy during the interpulse) is very similar in both cases, implying that the structure of the H-burning shell is not significantly affected by rotation. The same holds for the He-burning shell. The only noticeable difference is a small increase of the interpulse period in the rotating model. This can be ascribed to the higher Heflash luminosity and to the smaller 3DUP experienced by model B.

As far as the convective pulse is concerned, we report that its mass extent is slightly larger in the rotating case due to the presence of mixing at the convective borders of the pulse. The duration of the convective instability is also slightly longer if rotation is present since more nuclear fuel is engulfed in the pulse-driven convective zone.

As a conclusion, these tests show that the main factor influencing the structure of AGB stars is more the presence and the magnitude of the 3DUP than the supporting effect of rotational forces, at least as far as the angular velocity $\Omega$ remains subcritical. The effects of rotation are rather small and differences (interpulse duration, extent of the convective pulse, nuclear luminosities) account at most for a few percent, as far as the 3DUP amplitudes are similar. However, the influence of the mean molecular weight is unexpectedly large and can have a strong chemical impact on the role played by AGB stars on the chemical evolution of galaxies.

\section{4 s-Process Nucleosynthesis}

As diffusion operates below the convective envelope, protons are mixed with the carbon left by the pulse and when the temperature reaches $\sim 9 \times 10^{7} \mathrm{~K},{ }^{13} \mathrm{C}(\alpha, \mathrm{n}){ }^{13} \mathrm{~N}$ ignites. The outcome of hydrogen burning in this mixing zone depends strongly on the ratio of protons to carbon $\left(X_{\mathrm{p}} / X_{{ }^{12} \mathrm{C}}\right)$ (Goriely \& Mowlavi 2000). In layers where $X_{\mathrm{p}} / X_{{ }_{12} \mathrm{C}}>10^{-2}$, the $\mathrm{CN}$ cycle operates at equilibrium and ${ }^{14} \mathrm{~N}$ is mainly produced. Below this ${ }^{14} \mathrm{~N}$-rich layer, where the proton abundance is lower $\left(10^{-3}<X_{\mathrm{p}} / X_{{ }^{12} \mathrm{C}}<10^{-2}\right),{ }^{13} \mathrm{C}$ is preferentially formed.

The main characteristic of rotationally induced mixing is that diffusion remains at the base of the convective zone after the 3DUP proton injection. In this context, during the interpulse phase, ${ }^{14} \mathrm{~N}$ can migrate and eventually contaminate the adjacent ${ }^{13} \mathrm{C}$-rich layers. The large neutron capture cross section of nitrogen significantly reduces the s-process efficiency in the ${ }^{13} \mathrm{C}$ layer and prevents neutrons being captured by the iron seeds nuclei (see also Herwig et al. 2003). As a result, at the expense of s-elements, a substantial amount of ${ }^{14} \mathrm{C}$ is produced which later is responsible for the production of ${ }^{19} \mathrm{~F}$ in the convective pulse. To illustrate this point, we depict in Figure 4 the evolution of the main actors of the s-process. Initially, the strong diffusion coefficient triggers an efficient mixing of protons in the ${ }^{12} \mathrm{C}$-rich layers left by the pulse. After $\sim 500 \mathrm{yr}$, protons have almost reached their deepest extent $\left(M_{r} \sim 0.762784 \mathrm{M}_{\odot}\right)$ and start to react with ${ }^{12} \mathrm{C}$. Progressively a ${ }^{13} \mathrm{C}$ pocket builds up and $\sim 2000 \mathrm{yr}$ after the 3DUP proton injection, its abundance reaches a maximum value of $X_{{ }_{13} \mathrm{C}}=0.034$. After $\sim 3000 \mathrm{yr}$ the ${ }^{14} \mathrm{~N}$ pocket has largely engulfed the ${ }^{13} \mathrm{C}$ layers. As a result, the s-process nucleosynthesis is almost nonexistent with an overproduction of at most a factor of $\sim 3$ for the lightest s-elements (Fig. 5), in strong contrast with the overshoot model (case $D_{\text {rot }}=0$ ). This indicates that the present description of rotation-induced mixing is not favourable to the production of heavy nuclei by the s-process nucleosynthesis.

To investigate the sensitivity of the s-process nucleosynthesis on the mixing efficiency, we run a series of tests where we artificially increase and decrease the magnitude of the diffusion coefficient in our post processing code. However it must be emphasised that we did not recompute the stellar models with these modified diffusion coefficients. As a consequence, the initial chemical profiles that enter the nucleosynthesis code are not consistent. In particular, when we decrease the diffusion coefficient the initial proton profile which was computed with a larger value of $D_{\text {rot }}$ extends over a bigger mass range than it would in a consistent calculation. However, we keep this artifact as a way to simulate some kind of overshoot at 


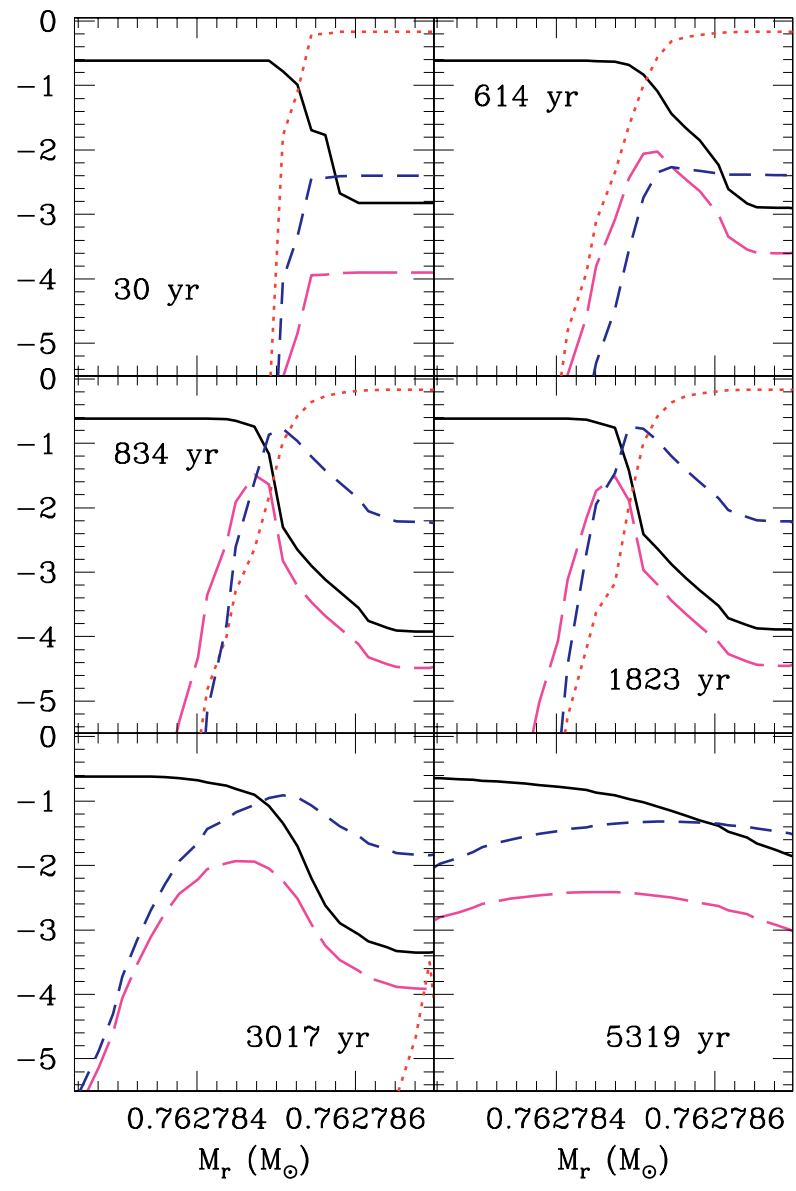

Figure 4 Evolution of the ${ }^{12} \mathrm{C}$ (solid), proton (dotted), ${ }^{14} \mathrm{~N}$ (shortdashed) and ${ }^{13} \mathrm{C}$ (long-dashed) profiles (in mass fraction) during the interpulse phase of the $3 \mathrm{M}_{\odot}$ rotating AGB models. The time corresponds to the time elapsed since the 3DUP.

the base of the convective envelope. On the other hand, if the diffusion coefficient is larger, the approximation is good because in this case the extent of the diffusion region will be larger than the one assumed here. The results of our tests are shown in Figure 5. Increasing the diffusion coefficient enhances the pollution of the ${ }^{13} \mathrm{C}$-rich layers by ${ }^{14} \mathrm{~N}$ and consequently weakens the s-process. In contrast, decreasing $D_{\text {rot }}$ prevents an efficient mixing of ${ }^{14} \mathrm{~N}$ within the ${ }^{13} \mathrm{C}$-rich layers and the s-process is therefore strengthened, provided diffusion is efficient enough to mix protons in the C-rich layer at the time of the 3DUP, by overshoot or gravity waves for instance. In the extreme case where $D_{\text {rot }}=0$, i.e. no rotational mixing is included just after the 3DUP, we recover the s-process distribution typical to the overshoot models (Goriely \& Mowlavi 2000). These results demonstrate that rotation can provide the conditions for the s-process nucleosynthesis, i.e. the production of the neutrons seeds. However the contamination of the ${ }^{13} \mathrm{C}$ layer by the poisonous ${ }^{14} \mathrm{~N}$ inhibits the neutron capture by the iron-peak nuclei and fails to synthesise heavy $(A>90)$ s-elements. This study also shows that the magnitude of the diffusion coefficient has a strong effects on the overproduction of s-elements. Finally, even if a correct neutron exposure can be obtained in this framework,

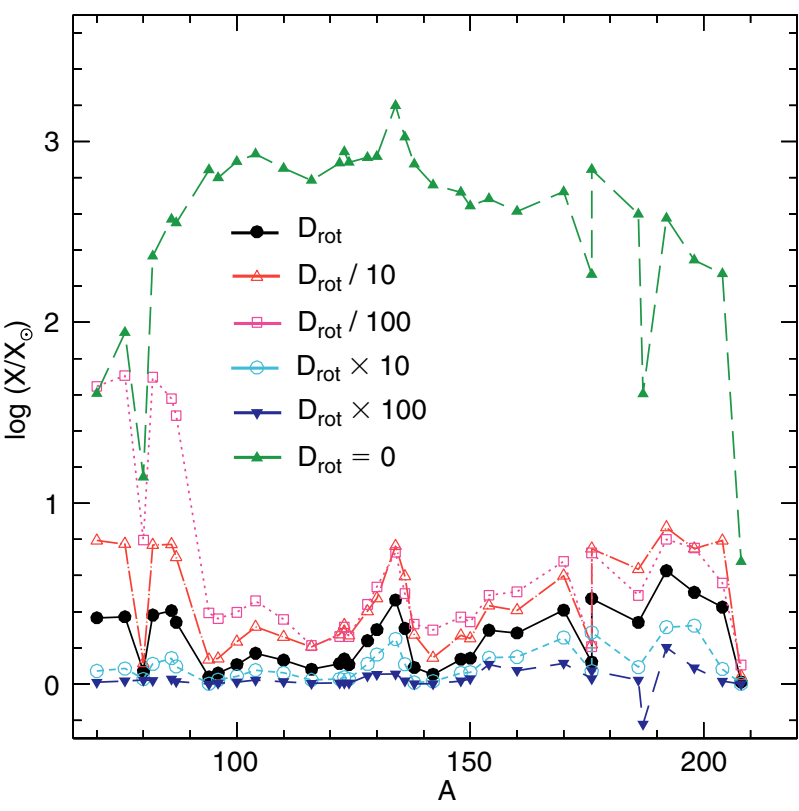

Figure 5 Overproduction factors of s-only elements obtained for the $3 \mathrm{M}_{\odot}$ rotating AGB models which experience the deep 3DUP $\left(f_{\mu}=0\right)$ as a function of the nuclear mass. The mass fraction of all species are summed over the same mass range and then normalised to solar. The distribution labelled $D_{\text {rot }}=0$ was computed with an initial proton profile in the $\mathrm{C}$-rich layer corresponding to a typical overshoot calculation.

the mass of the partial mixing zone is still too small to account for the observed abundances, as already discussed in Goriely \& Mowlavi (2000) and Herwig et al. (2003).

It should be noted that the derivation of the diffusion coefficient is still subject to large approximations and in particular our treatment of angular momentum transport does not take into account the advection term present in the theory developed by Zahn (1992). Its effect has been shown to be important during the main sequence of low-mass stars in particular in regions of strong gradients of mean molecular weight where it dominates the shearinduced mixing (Palacios et al. 2003). The role of this term is not easily predictable but may be important (see Sect. 3.2) and only full computations including this additional contribution would give a satisfying answer.

\section{Conclusion}

We analyse the impact of rotation on the structure and evolution of AGB stars. We show that the effects of centrifugal forces are rather weak in comparison to the effects induced by the mean molecular weight gradients. Omitting this contribution in the expression for the rotational mixing coefficient substantially increases the depth of the 3DUP. Our study of the s-process shows that rotation inhibits the production of s-process as a result of the contamination of the ${ }^{13} \mathrm{C}$ layers by ${ }^{14} \mathrm{~N}$. Depending on the magnitude of the diffusion coefficient, the distribution of s-process elements can be significantly different. These results suggest that rotation alone is not the main mechanism responsible for the s-process nucleosynthesis in AGB stars and indicate that rotational mixing can 
influence, and in particular reduce, the final enrichment in s-process elements.

\section{Acknowledgments}

L.S. acknowledges support from a European TMR 'Marie Curie' fellowship at ULB. S.G. is FNRS Research Associate.

\section{References}

Angulo, C., et al. 1999, Nucl. Phys. A, 656, 3

Brown, P. N., Hindmarsh, A. C., \& Petzold, L. R. 1994, SIAM J. Sci. Comp., 15, 1467

Brown, P. N., Hindmarsh, A. C., \& Petzold, L. R. 1998, SIAM J. Sci. Comp., 19, 1495

Denissenkov, P. A., \& Tout, C. A. 2003, MNRAS, 340, 722

Freytag, B., Ludwig, H.-G., \& Steffen, M. 1996, A\&A, 313, 497

Frost, C. A., \& Lattanzio, J. C. 1996, ApJ, 473, 383
Goriely, S. 1999, A\&A, 342, 881

Goriely, S., \& Mowlavi, N. 2000, A\&A, 362, 599

Goriely, S., \& Siess, L. 2001, A\&A, 378, L25

Heger, A., Langer, N., \& Woosley, S. E. 2000, ApJ, 528, 1033

Herwig, F. 2000, A\&A, 360, 952

Herwig, F., Blöcker, T., \& Schönberner, D. 1997, A\&A, 324, L81

Herwig, F., Lugaro, M., \& Langer, N. 2003, ApJ, 593, 1056

Jorissen, A., \& Goriely, S. 2001, Nuc. Phys., A688, 45c

Karakas, A. I., Lattanzio, J. C., \& Pols, O. R. 2002, PASA, 19, 515

Langer, N., Heger, A., Wellstein, S., \& Herwig, F. 1999, A\&A, 346, L37

Mowlavi, N. 1999, A\&A, 344, 617

Palacios, A., Talon, S., Charbonnel, C., \& Forestini, M. 2003, A\&A, 399,603

Pols, O. R., \& Tout, C. A. 2000, Mem. Soc. Astron. Italiana, 72, 299 Straniero, O., et al. 1997, ApJ, 478, 332

Takahashi, K., \& Yokoi, K. 1987, At. Data Nucl. Data Tables, 36,375

Zahn, J. P. 1992, A\&A, 265, 115 